\title{
Topological bulk lasing modes using an imaginary gauge field
}

\author{
Stephan Wong $\odot$ and Sang Soon Oh๑* \\ School of Physics and Astronomy, Cardiff University, Cardiff CF24 3AA, United Kingdom
}

(Received 21 December 2020; accepted 16 June 2021; published 9 July 2021)

\begin{abstract}
Topological edge modes, which are robust against disorders, have been used to enhance the spatial stability of lasers. Recently, it was revealed that topological lasers can be further stabilized using a topological phase in nonHermitian photonic topological insulators. Here we propose a procedure to realize topologically protected modes extended over a $d$-dimensional bulk by introducing an imaginary gauge field. This generalizes the idea of zeroenergy extended modes in the one-dimensional Su-Schrieffer-Heeger lattice into higher dimensional lattices, allowing a $d$-dimensional bulk mode that is topologically protected. Furthermore, we numerically demonstrate that the topological bulk lasing mode can facilitate high temporal stability superior to topological edge-mode lasers. Using an exemplified topological extended mode in the kagome lattice, we show that large regions of stability exist in its parameter space.
\end{abstract}

DOI: 10.1103/PhysRevResearch.3.033042

\section{INTRODUCTION}

In an attempt at ultimate control of the flow of light, photonic topological insulators (PTIs) [1] have enabled exciting devices such as unidirectional waveguides and topological lasers that are robust against perturbations and defects. In particular, the realization of robust topological optical systems has drawn attention for advanced photonics by reducing propagation loss in optical devices [2,3], quantum computers [4,5], photonic neural networks [6], and near-zero epsilon devices [7-9].

Recently, considerable effort has been made to study non-Hermitian PTIs by engaging topological edge modes to enable a lasing regime with optical nonlinearity $[10,11]$, distribution of gain and loss [12-15], and nonreciprocal couplings [16-18]. For example, the one-dimensional (1D) Su-Schrieffer-Heeger (SSH) model [19] has been utilized to generate edge states with gain and loss and implement topological lasing devices [11-13]. A cavity made of topologically distinct PTIs has been proposed to enhance the lasing efficiency by using unidirectional topologically protected edge modes $[15,16]$.

However, the edge-mode-based topological lasers are not appropriate for high-power lasers due to the localized nature of the edge modes and the possible breakdown of the constituting optical elements. As an alternative, topological bulk lasers have been proposed to achieve broad-area emission by using extended topological modes based solely on the parity symmetry at the $\Gamma$ point in a two-dimensional (2D) hexagonal

\footnotetext{
*ohs2@ cardiff.ac.uk

Published by the American Physical Society under the terms of the Creative Commons Attribution 4.0 International license. Further distribution of this work must maintain attribution to the author(s) and the published article's title, journal citation, and DOI.
}

cavity [17] or by using an imaginary gauge field in a 1D $\mathcal{P} \mathcal{T}$ symmetric SSH lattice to delocalize the zero-energy boundary mode over the 1D bulk [18].

Although the spatial stability of the topological lasing mode is guaranteed based on topological band theory, its temporal behavior is not necessarily stable due to the nonlinear nature of the laser rate equation [20]. Therefore, the temporal instability can further deteriorate the performance of lasing devices and it becomes important to study the dynamics and the temporal stability of the topological lasing modes [21].

In this work, we generalize the topological extended mode on the 1D SSH lattice to higher dimensional lattices. In particular, we demonstrate a topological extended mode on a 2D bulk by using a kagome lattice with a rhombus geometry and an imaginary gauge field. The topological bulk laser is studied with an alternating gain and loss distribution. We show that the topological extended mode lases and has large stable regions in its parameter space. We thus demonstrate that a phase-locked broad-area topological lasers can be realized in a two-dimensional (2D) kagome lattice.

The structure of this paper is as follows: In Sec. II, we recall the result in Ref. [18] where a topological extended mode is achieved using the 1D SSH lattice [19] along with an imaginary gauge field. Then Sec. III generalizes this result to higher dimensions. An explicit example is carried out on the kagome lattice with rhombus geometry. Section IV is dedicated to the study of the non-Hermitian kagome lattice in the active setting where the temporal dynamics of the topological protected mode is studied.

\section{EXTENDED TOPOLOGICAL MODE IN A 1D LATTICE}

Here, we briefly recall the procedure used to delocalize the topologically protected (zero-energy) mode in the SSH lattice, as presented in Ref. [18].

The SSH lattice, shown in Fig. 1(a), is a 1D lattice made of an array of $N_{s}$ sites. It has a unit cell composed of two sites 


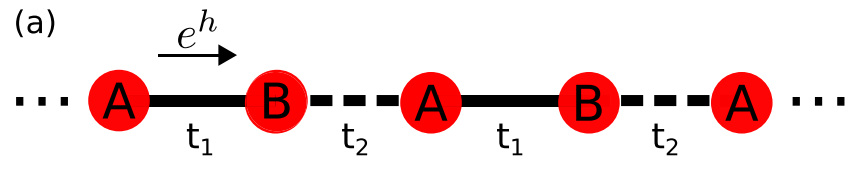

(b)

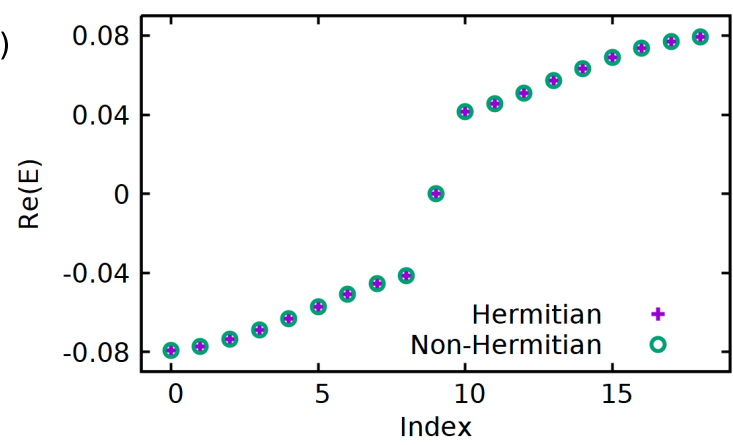

(c)

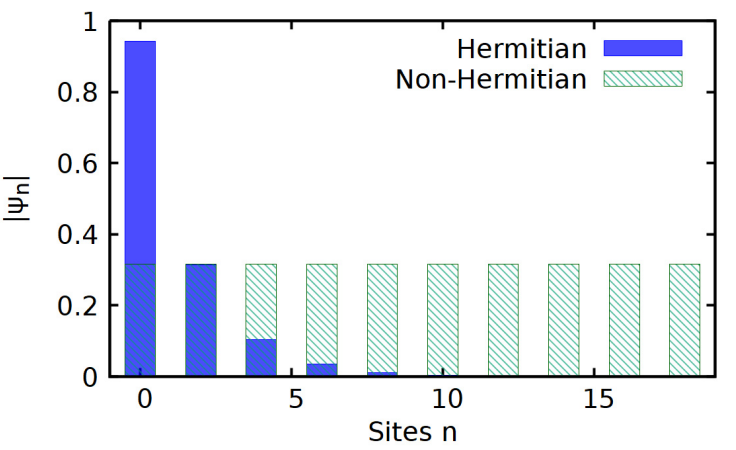

FIG. 1. (a) Schematic of a non-Hermitian SSH lattice made of an array of $N_{s}$ sites. The usual Hermitian SSH lattice corresponds to the case where $h=0$. (b) Spectrum of the finite-size SSH lattice in the Hermitian and non-Hermitian setting. The lattice starting from a site A and terminating at a site A. (c) Normalized field profile $\left|\psi_{n}\right|$ of the zero-energy mode from the finite-size SSH lattice in the Hermitian and non-Hermitian setting. The parameter are chosen such that $N_{s}=$ $19, E_{A}=E_{B}=0, t_{1}=0.02, t_{2}=0.06$, and $h=h_{0}$.

( $A$ and $B$ ) and the lattice is characterized by alternating intraand interunit cell couplings given by the real scalars $t_{1}$ and $t_{2}$, respectively.

In the non-Hermitian configurations, where an extended mode has been proposed [18,22], an imaginary gauge potential, $\mathcal{A}=-i h \boldsymbol{e}_{1}$, is introduced. In the presence of a gauge field, the Peierl's phase modifies the hopping terms by a factor: $e^{i \int \mathcal{A} \cdot \boldsymbol{d} \boldsymbol{l}}$, with $\boldsymbol{d} \boldsymbol{l}=d x_{i} \boldsymbol{e}_{\boldsymbol{i}}$, the direction of the hopping. The couplings are therefore modified and become asymmetric. Note that the usual prefactors are here absorbed in $\mathcal{A}$, or equivalently in $h$. The coupling constants get a $e^{h}$ factor term when hopping from left to right and a $e^{-h}$ factor term when hopping from right to left. The coupled-mode equations are then written as

$$
\begin{aligned}
& i \frac{d a_{n}}{d t}=E_{A} a_{n}+t_{1} e^{-h} b_{n}+t_{2} e^{h} b_{n-1}, \\
& i \frac{d b_{n}}{d t}=E_{B} b_{n}+t_{1} e^{h} a_{n}+t_{2} e^{-h} a_{n+1},
\end{aligned}
$$

with $a_{n}$ and $b_{n}$ being the modal amplitudes on the $\mathrm{A}$ and $\mathrm{B}$ sites at the $n$th unit cell, respectively. $E_{\sigma}$ is the on-site energy on the site $\sigma$.

For a finite system, the introduction of the imaginary gauge field will not affect the spectrum [22]. This is made explicit here because the system of equations above can be solved with a suitable gauge transformation:

$$
\begin{gathered}
a_{n}=e^{2 h n} \tilde{a}_{n}, \\
b_{n}=e^{2 h n} e^{-2 h} \tilde{b}_{n},
\end{gathered}
$$

where $a_{n}$ and $b_{n}$ are solutions of the coupled-mode equations if $\tilde{a}_{n}$ and $\tilde{b}_{n}$ are solutions of the Hermitian SSH coupled-mode equations, namely, when $h=0$.

For a SSH lattice starting and terminating on an A site, it is known that the zero-energy mode of the Hermitian SSH lattice reads

$$
\begin{gathered}
\tilde{a}_{n}=\tilde{r}^{n} \tilde{a}_{0}, \\
\tilde{b}_{n}=0, \forall n,
\end{gathered}
$$

where $\tilde{r}=-\frac{t_{1}}{t_{2}}$ defined as $\tilde{a}_{n+1}=\tilde{r} \tilde{a}_{n}$ and satisfies the destructive interference condition on the B sites $t_{1}+\tilde{r} t_{2}=0$.

The solution for the non-Hermitian SSH lattice is then written as

$$
\begin{gathered}
a_{n}=r^{n} a_{0}, \\
b_{n}=0, \forall n,
\end{gathered}
$$

where $r=-\frac{t_{1}}{t_{2}} e^{2 h}$ defined as $a_{n+1}=r a_{n}$ and satisfies the destructive interference condition on the $\mathrm{B}$ sites $t_{1} e^{h}+r t_{2} e^{-h}=$ 0 .

The main effect of this imaginary gauge field is to change the localization property of the modes without affecting the spectrum. In particular, one can delocalize the topological protected edge mode over the whole 1D bulk, while keeping its topological protection from the chiral symmetry of the Hermitian topologically protected (zero-energy) mode. The exponentially increasing or decaying factor is removed by appropriately choosing the gauge field $h$ :

$$
h=h_{0}:=-\frac{1}{2} \ln \left(\frac{t_{1}}{t_{2}}\right) .
$$

Figure 1(b) shows that the spectrum of the finite-size Hermitian and non-Hermitian SSH lattice are indeed identical. However, Fig. 1(c) shows, for $t_{1}<t_{2}$, that the field profile $\left|\psi_{n}\right|$ of the zero-energy mode, which is localized on the left edge for the Hermitian case, is extended over the 1D bulk for the non-Hermitian case.

Finally, it is worth noting that although the zero-energy mode is topologically protected, its localization property depends on the coupling constants and is therefore sensitive to their perturbations. However, for reasonably small perturbations, i.e., small enough so that the band gap does not close, the delocalization is not destroyed: The amplitudes remain of the same order of magnitude over the bulk but are simply not equal anymore [see Fig. 6(f)]. 
(a)

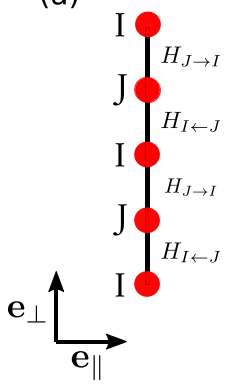

(b)

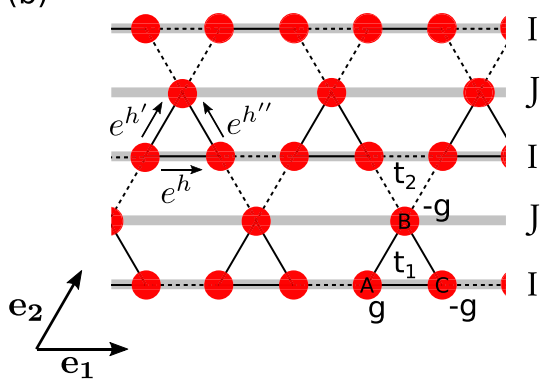

FIG. 2. (a) Schematic of a $d$-dimensional lattice in a quasi1D lattice made of an array of $N_{s}(d-1)$-dimensional lattices. (b) Schematic of the kagome lattice drawn in the quasi-1D lattice formalism. The solid and dashed lines denote the intracell $\left(t_{1}\right)$ and intercell couplings $\left(t_{2}\right)$, respectively. $e^{h}, e^{h^{\prime}}, e^{h^{\prime \prime}}$ correspond to the imaginary gauge field introduced for delocalizing the topological mode. in the non-Hermitian kagome lattice. $g(-g)$ is the gain (loss) considered for the laser setting.

\section{EXTENDED TOPOLOGICAL MODE IN A $\boldsymbol{d}$-DIMENSIONAL LATTICE: EXAMPLE ON THE KAGOME LATTICE}

We now generalize this notion of delocalized (or extended) topological mode over a whole $d$-dimensional bulk.

\section{A. General framework}

The strategy follows the previous section, namely to find an exact solution of the (topologically protected) boundary state, and then use non-Hermiticity to change the localization property of the chosen mode.

In order to find an exact solution, the procedure follows Refs. [23,24]. One needs to consider a $d$-dimensional lattice as a stack of $(d-1)$-dimensional lattices $\left(\boldsymbol{e}_{\|}\right.$direction) and with an open boundary condition (OBC) in the remaining one-dimensional ( $\boldsymbol{e}_{\perp}$ direction) boundary [23-25] (see Fig. 2 for the example of the kagome lattice). The lattice can then be considered as a quasi-1D lattice with the unit cell composed of two lattice sites, $I$ and $J$, except that here the lattice sites represent $(d-1)$-dimensional lattices, as shown in Fig. 2. Additional conditions are assumed such that the quasi-1D lattice needs to start and terminate on the same lattice site, and we forbid direct hopping between the $I$ lattice sites [23,24,26]. Therefore, the lattice naturally supports the exact disappearance of the modal amplitude of $n$ modes on the $J$ lattice sites, with $n$ being the number of degrees of freedom on the $I$ lattice site.

From the quasi-1D formalism, the coupled-mode equation is conveniently written as

$$
i \frac{d \Psi}{d t}=H_{\text {lattice }} \Psi
$$

where $\Psi=\left(\psi_{I, 0}, \psi_{J, 0}, \ldots, \psi_{I, N}\right)^{T}$ with $\psi_{I, n}$ and $\psi_{J, n}$ being the modal amplitudes on the $I$ and $J$ lattice sites in the $n$th stacked unit cell, respectively. $N$ is the index of the last unit cell.

The Hamiltonian of the lattice reads

$$
H_{\text {lattice }}=\left(\begin{array}{cccc}
H_{I} & H_{I \leftarrow J} & 0 & \cdots \\
\tilde{H}_{I \leftarrow J}^{\dagger} & H_{J} & \tilde{H}_{J \rightarrow I}^{\dagger} & \ldots \\
0 & H_{J \rightarrow I} & H_{I} & \ldots \\
\vdots & \vdots & \vdots & \ddots
\end{array}\right)
$$

with $H_{I}$ and $H_{J}$ being the Hamiltonian of the lattice $I$ and $J$, respectively. $H_{I \leftarrow J}$ and $H_{J \rightarrow I}$ are, respectively, the intra- and interunit cell couplings between the $I$ and $J$ lattices. For the Hermitian case, $\tilde{H}_{I \leftarrow J}^{\dagger}=H_{I \leftarrow J}^{\dagger}$ and $\tilde{H}_{J \rightarrow I}^{\dagger}=H_{J \rightarrow I}^{\dagger}$.

For the general $d$-dimensional lattice, the eigenvalue problem $H_{\text {lattice }} \Psi=E \Psi$ yields, for $n=1, \ldots, N$,

$$
\begin{aligned}
& H_{I, k} \psi_{I, n}+H_{I \leftarrow J} \psi_{J, n}+H_{J \rightarrow I} \psi_{J, n-1}=E \psi_{I, n}, \\
& H_{J, k} \psi_{J, n}+\tilde{H}_{I \leftarrow J}^{\dagger} \psi_{I, n}+\tilde{H}_{J \rightarrow I}^{\dagger} \psi_{I, n+1}=E \psi_{J, n} .
\end{aligned}
$$

The condition for destructive interference on the $J$ lattices, $\psi_{J, n}=0$, is given by

$$
\tilde{H}_{I \leftarrow J}^{\dagger} \psi_{I, n}^{(i)}+\tilde{H}_{J \rightarrow I}^{\dagger} \psi_{I, n+1}^{(i)}=0 .
$$

From Eq. (12), the solution with vanishing amplitude on the $J$ lattice therefore gives the additional condition

$$
H_{I} \psi_{I, n}=E \psi_{I, n} ;
$$

namely $\psi_{I, n}$ must be an eigenmode of the Hamiltonian on the lattice $I$, labeled $\psi_{I, n}^{(i)}$, with corresponding energy $E=E_{I}^{(i)}$.

Since we are looking at edge states, one can ask for solutions which exponentially decay or increase, or equivalently solutions that satisfy $[23,25]$

$$
\psi_{I, n+1}^{(i)}=r_{i} \psi_{I, n}^{(i)}
$$

with $r_{i}$ being a scalar term representing the decaying amplitudes of the mode inside the quasi-1D lattice.

The boundary state solution of the Hamiltonian $H_{\text {lattice }}$ with energy $E_{I}^{(i)}$ is therefore of the form

$$
\psi_{I, n}^{(i)}=r_{i}^{n} \psi_{I, 0}^{(i)}
$$

with $r_{i}$ satisfying the destructive interference condition on the $J$ lattice:

$$
\tilde{H}_{I \leftarrow J}^{\dagger}+r_{i} \tilde{H}_{J \rightarrow I}^{\dagger}=0 .
$$

In summary, the modes with eigenenergy $E=E_{I}^{(i)}$, such that $H_{I} \psi_{I}^{(i)}=E_{I} \psi_{I}^{(i)}$, are the modes which are exponentially localized on one edge and with nonvanishing amplitudes only on the lattice sites $I$, with adjacent $\pi$-phase difference and with mode distribution corresponding to $\psi_{I}^{(i)}$ on the lattice site I.

The delocalization of the edge modes is realized by introducing an imaginary gauge field such that $\left|r_{i}\right|=1$. The non-Hermiticity allows the change of the localization properties while keeping the spectrum unchanged.

\section{B. Extended topological mode in 2D kagome lattice}

As a concrete example, we will look at the case of the kagome lattice as shown in Fig. 2. The Appendix provides additional examples with the Lieb and the 2D SSH lattices. The kagome lattice is characterized by unit cells composed of 


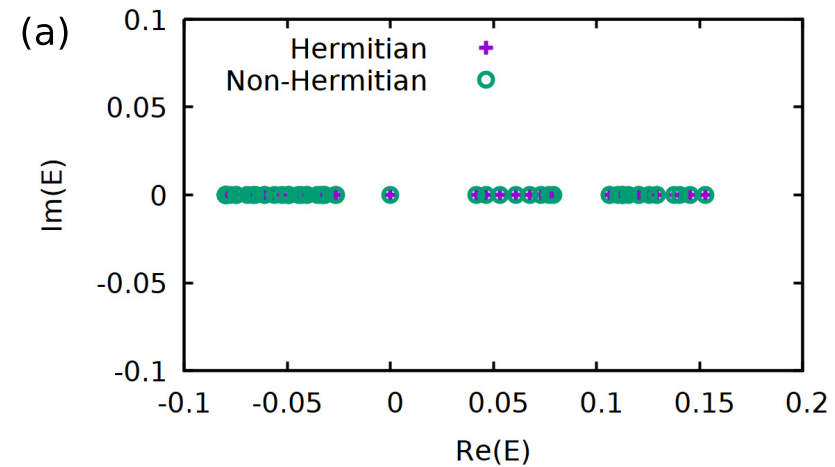

(b)

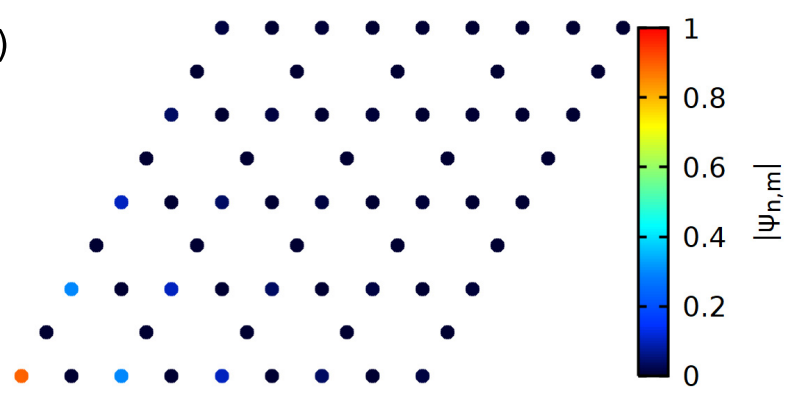

(c)

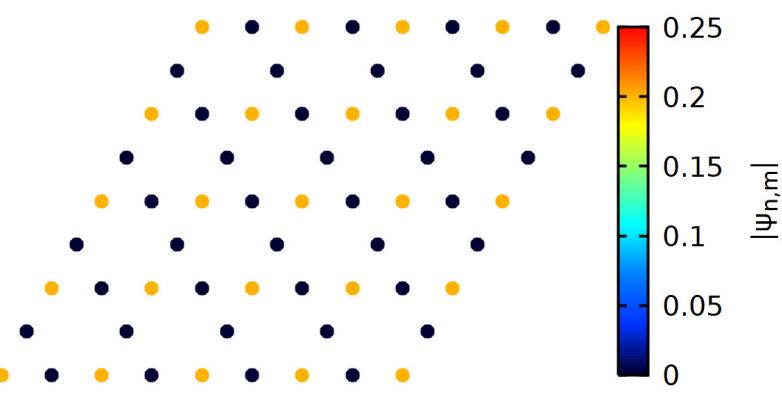

FIG. 3. (a) Spectrum of the kagome lattice in the rhombus geometry in the Hermitian and non-Hermitian setting. The normalized field profile $\left|\psi_{n, m}\right|$ of the zero-energy mode of the kagome lattice in the rhombus geometry is plotted in panel (b) for the Hermitian case and in panel (c) for the non-Hermitian case. Here, there are $N_{s}=9$ sites both in the $I$ lattice and the quasi-1D lattice. $t_{1}=0.02, t_{2}=$ 0.06 . The Hermitian setting corresponds to the case $h=h^{\prime}=h^{\prime \prime}=0$, whereas the non-Hermitian setting is for $h=h^{\prime}=h_{0}, h^{\prime \prime}=0$.

three sites, $\mathrm{A}, \mathrm{B}$, and $\mathrm{C}$, and the coupling strengths between sites are different for intraunit cell $\left(t_{1}\right)$ and interunit cell $\left(t_{2}\right)$ couplings.

We will specifically look at the topological zero-energy corner mode present in the rhombus geometry of the kagome lattice $[27,28]$ [see Fig. 3(b) for the geometry of the lattice]. Figure 3(a) shows the spectrum of the kagome lattice in the rhombus geometry. Figure 3(b) plots the normalized field distribution $\left|\psi_{n, m}\right|$ of the zero-energy mode for $t_{1}<t_{2}$. This shows that the mode is indeed localized on the bottom-left corner, with vanishing amplitudes on the $\mathrm{B}$ and $\mathrm{C}$ sites as explained later.

Applying the previous quasi-1D formalism to the kagome lattice in the rhombus geometry, we have $H_{I}=H_{\mathrm{SSH}}$, $H_{J}=\operatorname{diag}\left(E_{B}, \ldots, E_{B}\right)$. The intra- and interstacked lat- tice couplings are $\left(N_{s} \times 1\right)$ matrices given respectively by $\tilde{H}_{I \leftarrow J}^{\dagger}=H_{I \leftarrow J}^{\dagger}=\left(t_{1}, t_{1}, \ldots, t_{1}, t_{1}\right)^{T}$ and $\tilde{H}_{J \rightarrow I}^{\dagger}=H_{J \rightarrow I}^{\dagger}=$ $\left(t_{2}, t_{2}, \ldots, t_{2}, t_{2}\right)^{T} . N_{s}$ is the number of sites on the $I$ lattices.

The rhombus geometry is interesting since in the $\boldsymbol{e}_{\|}$direction, the $I$ lattices, which are equivalent to the SSH lattice, start with and are terminated by the same site (here site A). In this configuration, we can see the zero-energy corner mode is related to the topological zero-energy mode present in the chiral symmetric SSH lattice. The zero-energy corner mode can be seen as the boundary state of the kagome lattice with eigenenergy $E_{I}^{(i)}=E_{I}^{(0)}=0$ and can be analytically written as [24]

$$
\psi_{I, m}^{(0)}=r_{0,2}^{m} \psi_{I, 0}^{(0)}
$$

with $\left[\psi_{I, 0}^{(0)}\right]_{n}=r_{0,1}^{n} a_{0,0}$ being the $n$th component of the zeroenergy mode $\psi_{I, 0}^{(0)}$ of the SSH lattice where the interference conditions [Eq. (18)] give $r_{0,1}=-\frac{t_{1}}{t_{2}}$ and $r_{0,2}=-\frac{t_{1}}{t_{2}} \cdot a_{n, m}$ is the modal amplitude on the A site at the $n$th unit cell in the $m$ th lattice $I$. As expected, the analytical expression show that choosing different intra- and interunit cell coupling constants, $t_{1}<t_{2}$ or $t_{1}>t_{2}$, the zero mode is exponentially localized, respectively, on the bottom-left or upper-right edge of the SSH lattice with vanishing amplitudes on the $\mathrm{B}$ and $\mathrm{C}$ sites.

We use an imaginary gauge field to change the localization property of this corner mode [18,22]. Figure 2(b) sketches the gauge potential considered where $e^{h}, e^{h^{\prime}}$, and $e^{h^{\prime \prime}}$ represent the phase factor in the couplings between the sites $\mathrm{A}$ and $\mathrm{B}, \mathrm{A}$ and $\mathrm{C}$, and $\mathrm{B}$ and $\mathrm{C}$, respectively. Conditions on $h, h^{\prime}$, and $h^{\prime \prime}$ are imposed given an imaginary gauge field $\mathcal{A}=\left(\mathcal{A}_{1}, \mathcal{A}_{2}\right)$. From the Peierls phase corresponding to $e^{h}$, we have $\mathcal{A}_{1}=$ $-i h$, with $\boldsymbol{d} \boldsymbol{l}=\boldsymbol{e}_{\mathbf{1}}$. Similarly, $e^{h^{\prime}}$ gives $\mathcal{A}_{2}=-i h^{\prime}$, using $\boldsymbol{d} \boldsymbol{l}=$ $\boldsymbol{e}_{2}$. These two conditions on $\mathcal{A}$ mean that for $e^{h^{\prime \prime}}$ we must have

$$
h^{\prime \prime}=-h+h^{\prime}
$$

using $d l=e_{2}-e_{1}$. With the imaginary gauge field, the coupling matrices are then modified, and particularly $\tilde{H}_{I \leftarrow J}^{\dagger}=\left(t_{1} e^{h^{\prime}}, t_{1} e^{h^{\prime \prime}}, \ldots, t_{1} e^{h^{\prime}}, t_{1} e^{h^{\prime \prime}}\right)$ and $\tilde{H}_{J \rightarrow I}^{\dagger}=$ $\left(t_{2} e^{-h^{\prime}}, t_{2} e^{-h^{\prime \prime}}, \ldots, t_{2} e^{-h^{\prime}}, t_{2} e^{-h^{\prime \prime}}\right)$. The interference conditions now yield

$$
r_{0,1}=-\frac{t_{1}}{t_{2}} e^{2 h}
$$

and

$$
r_{0,2}=-\frac{t_{1}}{t_{2}} e^{2 h^{\prime}}
$$

Delocalization over the $\boldsymbol{e}_{\mathbf{1}}$ direction is achieved by requiring $\left|r_{0,1}\right|=1$, namely choosing $h=h_{0}$. Similarly, delocalization over the $\boldsymbol{e}_{2}$ direction is realized with $h^{\prime}=h_{0}$ so that $\left|r_{0,2}\right|=1$. One can notice that there is no further condition on $h^{\prime \prime}$ to delocalize the mode in the quasi-1D lattice. This is because of the vanishing amplitudes on the $\mathrm{B}$ and $\mathrm{C}$ sites. Figures 4(a) and 4(b) show the normalized field profile $\left|\psi_{n, m}\right|$ of the zero-energy mode using $h=h_{0}, h^{\prime}=h^{\prime \prime}=0$, and $h^{\prime}=h_{0}$, $h=h^{\prime \prime}=0$, respectively. In Fig. 4(a), the mode is localized on the bottom edge while being extended over the $\boldsymbol{e}_{1}$ direction. On the other hand, Fig. 4(b) shows that the mode is localized on the left edge while being extended along the $\boldsymbol{e}_{2}$ direction. It is worth noting that for the values of $h, h^{\prime}$, and $h^{\prime \prime}$ chosen 

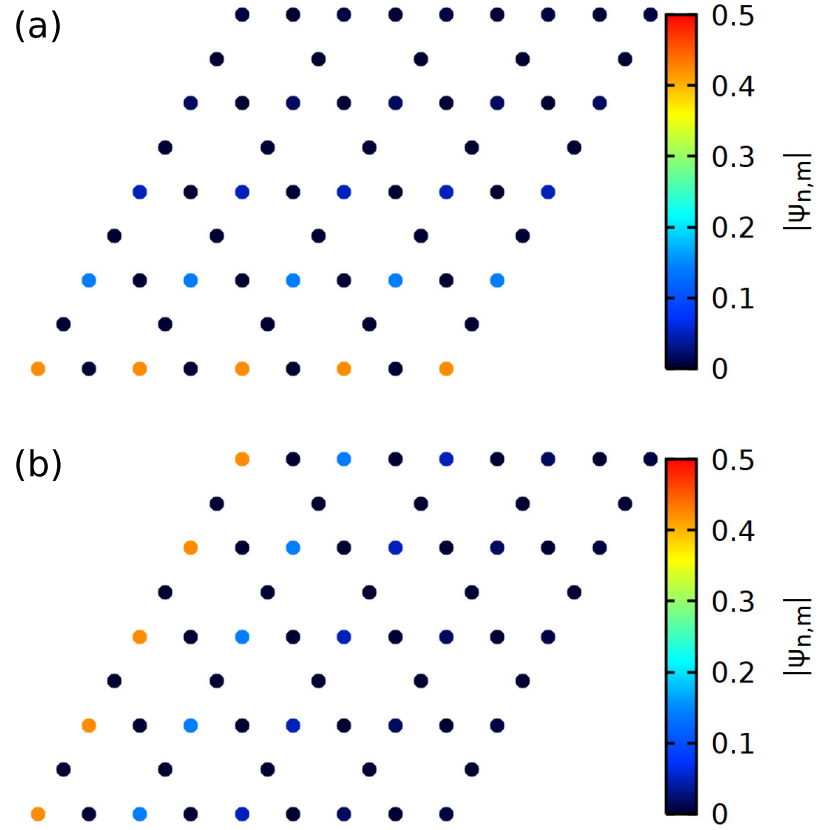

FIG. 4. Normalized field profile $\left|\psi_{n, m}\right|$ of the zero-energy mode of the kagome lattice in the rhombus geometry with (a) $h=h_{0}, h^{\prime}=$ $h^{\prime \prime}=0$ and (b) $h^{\prime}=h_{0}, h=h^{\prime \prime}=0$. The other parameters are the same as in Fig. 3.

for drawing Fig. 4, the spectrum has been changed compare to the Hermitian case.

Provided Eq. (20) holds, the introduction of the imaginary gauge field will only affect the localization property of the mode while keeping the spectrum unchanged. Therefore, in this case, the condition $\left|r_{0, i}\right|=1$ does not correspond to band touching with the edge band and the bulk band [23].

Combining the two results obtained above for the delocalization of the zero-energy mode, we have $h=h^{\prime}=h_{0}$ and, from Eq. (20), $h^{\prime \prime}=0$. Figure 3(a) shows that the numerically calculated spectrum of the kagome lattice in the rhombus geometry does not change when the imaginary gauge is introduced. Figure 3(c) plots the normalized field distribution of the zero-energy mode using $h=h^{\prime}=h_{0}$ and $h^{\prime \prime}=0$. This topologically protected zero-energy mode is therefore extended over the whole bulk of the kagome lattice: It is a topological bulk mode in the 2D kagome lattice.

Generalization to higher dimensional lattices can be achieved using a similar procedure, namely starting by delocalizing a topologically protected mode over a lowdimensional bulk and repeating this step with the higher dimension.

\section{LASING IN THE NON-HERMITIAN KAGOME LATTICE}

The peculiarities of this extended topological mode are its vanishing amplitudes on the $\mathrm{B}$ and $\mathrm{C}$ sites, but most of all, that it is topologically protected over a $2 \mathrm{D}$ bulk and has a $\pi$-phase difference between nonvanishing sites. This hints at the possibility to realize phase-locked broad-area topological lasers in 2D lattices.
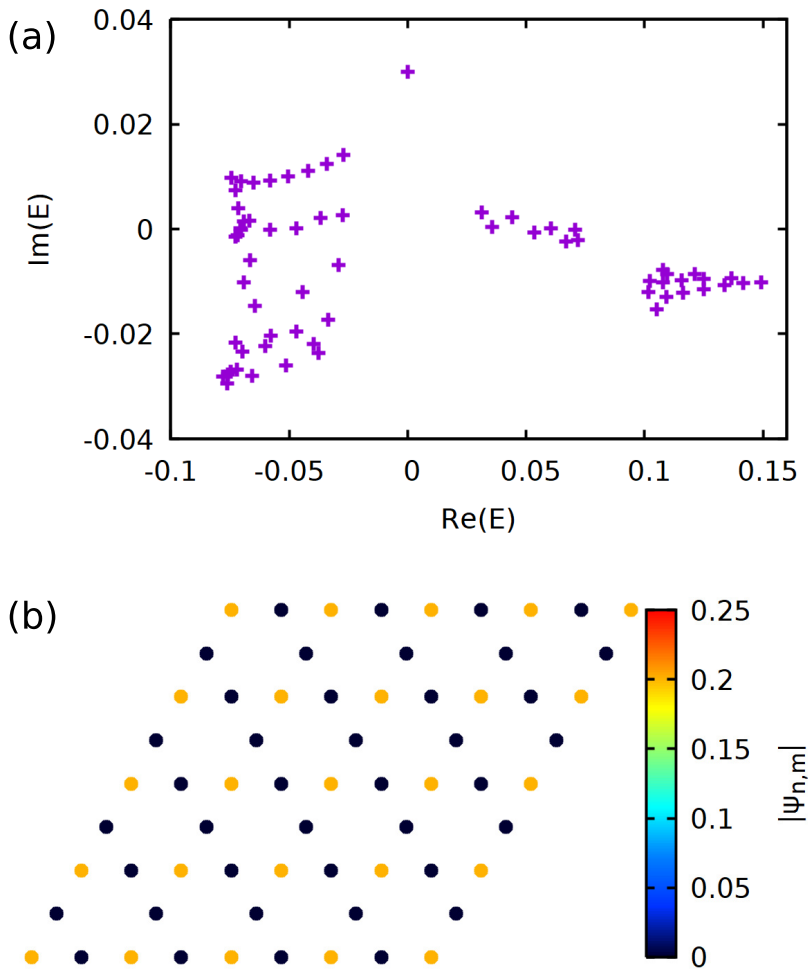

FIG. 5. (a) Spectrum of the active kagome lattice in the rhombus geometry. (b) Normalized field profile $\left|\psi_{n, m}\right|$ of the zero-energy mode. We have $N_{s}=9$ sites both in the $I$ lattice and the quasi-1D lattice, $t_{1}=0.02, t_{2}=0.06, g=0.03$ and $h=h^{\prime}=h_{0}, h^{\prime \prime}=0$.

\section{A. An active and non-Hermitian kagome lattice}

The design presented above can be extended to present a topological laser by considering optical pumping of semiconductor ring resonators with gain $(g)$ and loss $(-g)$, as the sites. The imaginary gauge field can possibly be made using auxiliary ring resonators with different losses strength between the upper and lower half perimeter of the ring [18]. The values of the imaginary gauge field $h, h^{\prime}$, and $h^{\prime \prime}$ would then mainly depend on the losses contrast by depositing lossy materials with different thickness on top of the auxiliary rings or using different materials [11,13,29,30].

The gain and loss configuration is chosen based on the $\mathrm{SSH}$ sublattices since we are looking for topological modes in the kagome lattice that originate from the topological mode in the $\mathrm{SSH}$ sublattice. In the literature, it is well known that the SSH lattice in a $\mathcal{P} T$-symmetric configuration [11,12] (gain on $\mathrm{A}$ sites, $E_{A}=i g$, and loss on $\mathrm{C}$ sites, $E_{C}=-i g$ ) can preserve the topological protection of the zero energy. In particular, if the SSH lattice is in the unbroken $\mathcal{P} T$-symmetric phase, i.e., $g<\left|t_{2}-t_{1}\right|$, then the gap does not close in the presence of gain or loss and the zero-energy mode remains well separated from the bulk $[11,14]$. When the energy is complex, we refer to the zero energy as the real part of the energy being zero.

Since the only nonvanishing terms of the extended topological mode are located in the A sites [as shown in Eq. (19) and Fig. 3(c)], we would like lasing to come from these sites. Therefore, we set gain on site $\mathrm{A}, E_{A}=i g$, and lossy 

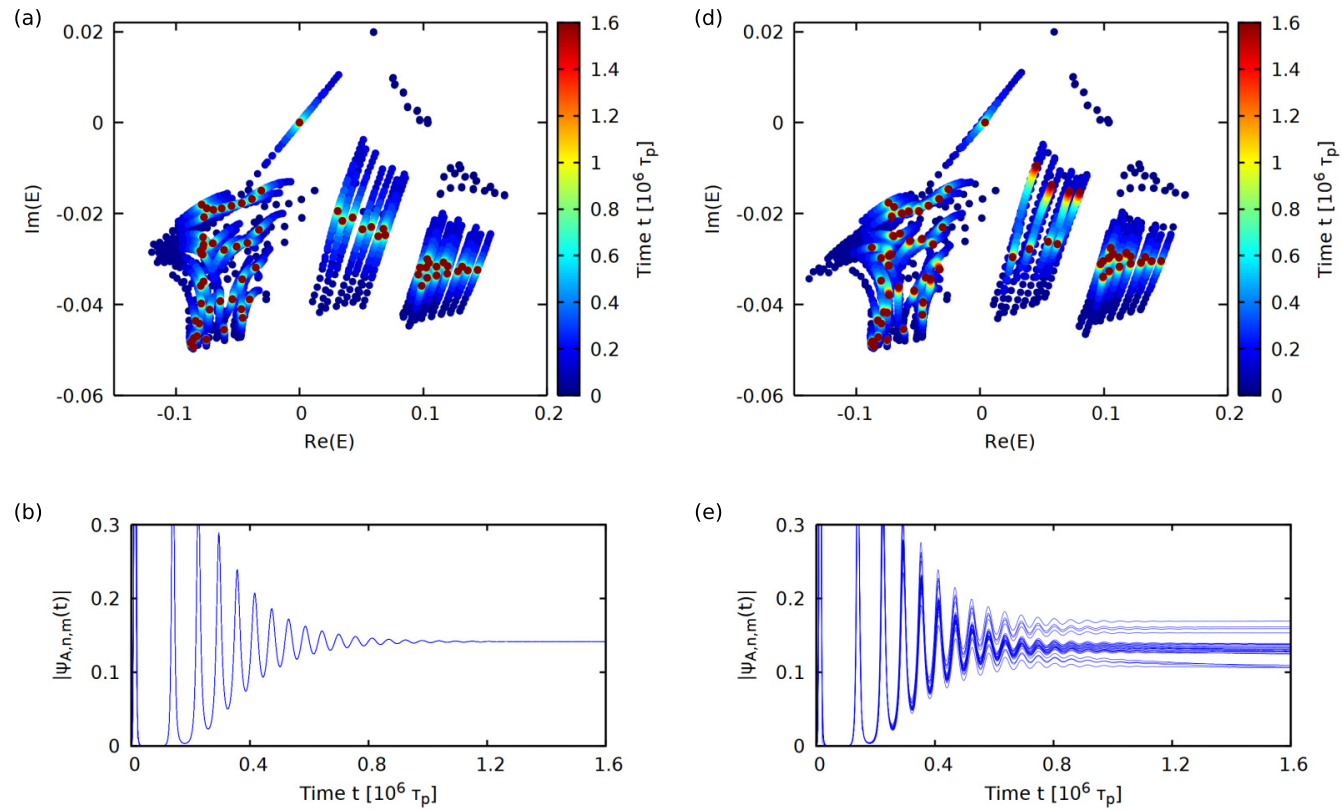

(e)
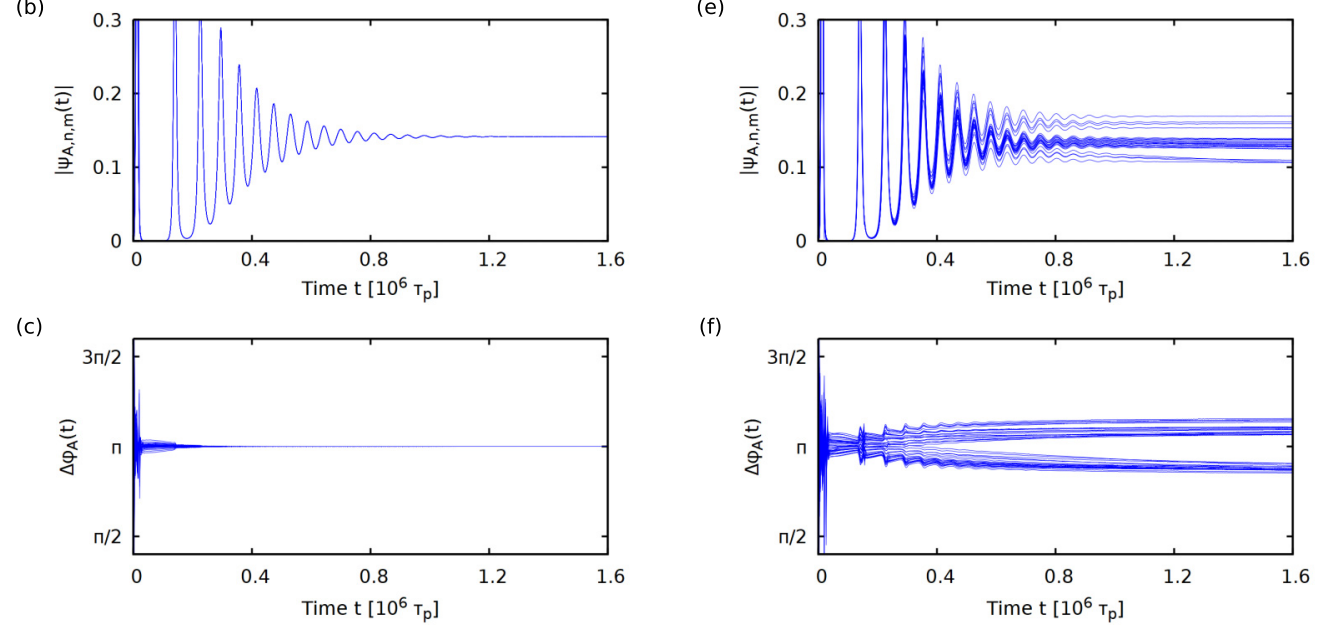

FIG. 6. Time evolution of (a) the instantaneous spectrum of the kagome lattice in rhombus geometry, (b) the amplitudes of all the $A$ sites, and (c) the phase differences between the adjacent $A$ sites when there is no disorder. Similarly for panels (d), (e), and (f) when asymmetric disorders is introduced. The parameters used are $\tau_{p}=40 \mathrm{ps}, \alpha=3, \tau_{s}=80 \mathrm{~ns}, p_{A}=0.02, g_{B} \tau_{p}=g_{C} \tau_{p}=0.05, \delta_{B} \tau_{p}=\delta_{C} \tau_{p}=0.01, t_{1} \tau_{p}=$ $0.02, t_{2} \tau_{p}=0.06$.

rings on the $\mathrm{B}$ and $\mathrm{C}$ sites, $\left(E_{B}=E_{C}=-i g\right)$, as shown in Fig. 2(b).

Figure 5(a) shows the spectrum in the complex plane, numerically calculated with $E_{A}=i g, E_{B}=E_{C}=-i g, h=$ $h^{\prime}=h_{0}$, and $h^{\prime \prime}=0$. This demonstrates that the zero mode is present. Because of the gain on the A sites, i.e., where the zero-energy mode is nonvanishing, the zero-energy mode has higher gain compared to the other modes. Figure 5(b) displays the normalized field profile $\left|\psi_{n, m}\right|$ of the zero-energy mode. This shows that although active design has been considered, the delocalization of the zero-energy mode is not altered.

\section{B. Temporal dynamics of the zero-energy mode}

Time-domain modeling of the mode dynamics is essential for determining whether the lasing mode is stable. In the frequency analysis, we have shown it is possible to have an active non-Hermitian kagome lattice with an extended topological mode. However, the previous analysis provides only a simple physical model of the active nonHermitian kagome lattice. Indeed, it has been shown that temporal instabilities in the laser array may prevent phase locking and reduce the laser quality or even dominate and suppress the topological signature of the corresponding lasing mode $[18,20]$.

We consider the laser rate equation for modeling the gain in the active rings $\mathrm{A}[18,31,32]$. Optical pumping are assumed to be small enough to leave the lossy ring resonators (rings $\mathrm{B}$ and $\mathrm{C}$, and auxiliary rings) in the linearized regime below their threshold point. Linear losses are thus chosen, and the losses and imaginary gauge field are considered to be carrier independent. The laser rate equation in the kagome lattice, with $h=h^{\prime}=h_{0}$ and $h^{\prime \prime}=0$, is then

$$
\begin{aligned}
i \frac{d a_{n, m}}{d t}= & \frac{1}{\tau_{p}}(1-i \alpha) Z_{n, m} a_{n, m}+t_{1} e^{-h_{0}} b_{n, m}+t_{1} e^{-h_{0}} c_{n, m} \\
& +t_{2} e^{h_{0}} b_{n, m-1}+t_{2} e^{h_{0}} c_{n-1, m} \\
i \frac{d b_{n, m}}{d t}= & -i g_{B} b_{n, m}+t_{1} e^{h_{0}} a_{n, m}+t_{1} c_{n, m} \\
& +t_{2} e^{-h_{0}} a_{n, m-1}+t_{2} c_{n-1, m+1} \\
i \frac{d c_{n, m}}{d t}= & -i g_{C} c_{n, m}+t_{1} e^{h_{0}} a_{n, m}+t_{1} b_{n, m} \\
& +t_{2} e^{-h_{0}} a_{n+1, m}+t_{2} b_{n+1, m-1} \\
\tau_{s} \frac{d Z_{n, m}}{d t}= & p_{A}-Z_{n, m}-\left(1+2 Z_{n, m}\right)\left|a_{n, m}\right|^{2}
\end{aligned}
$$



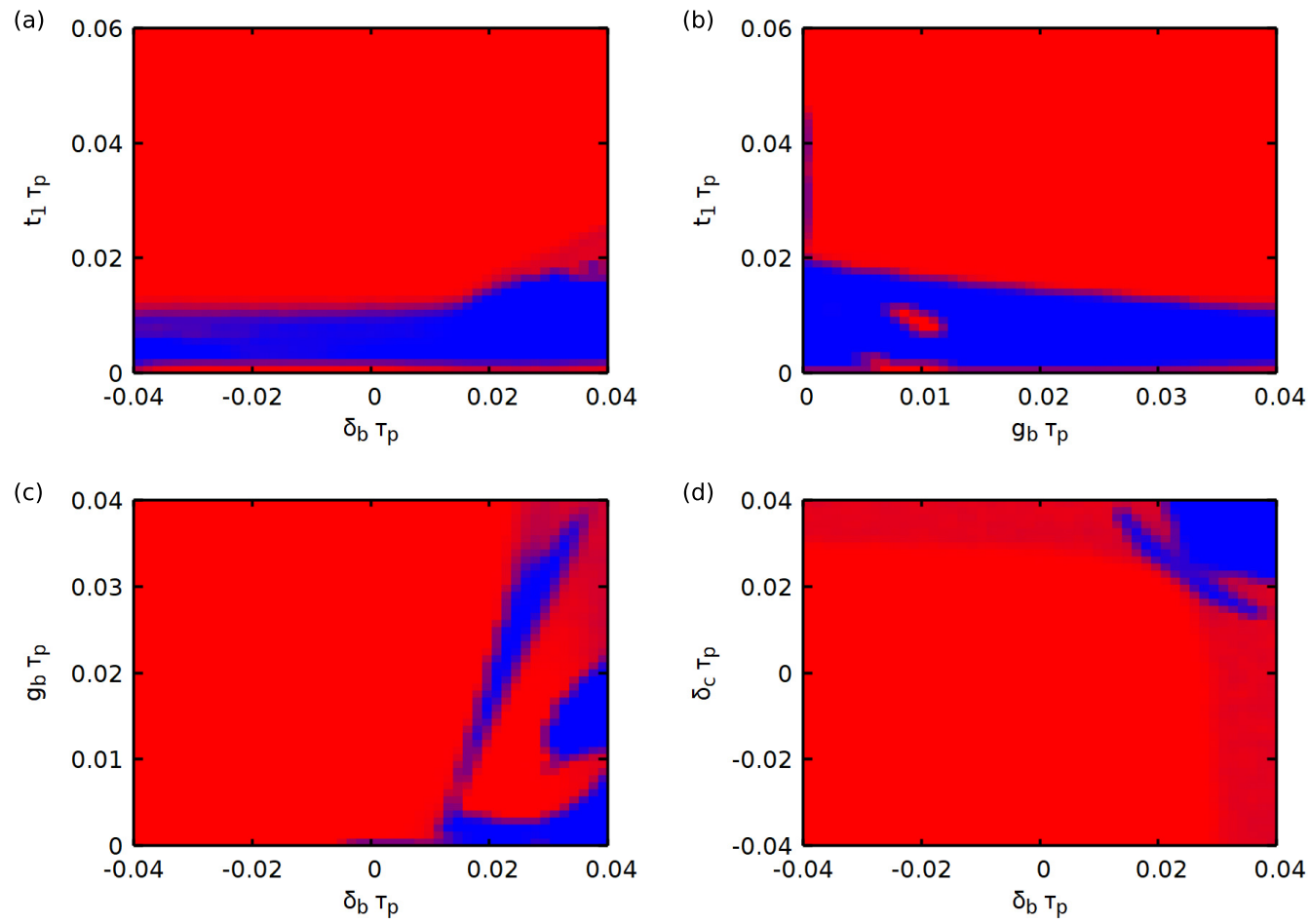

Probability of stable state

FIG. 7. Stability diagrams of the topological extended mode. The color corresponds to the probability of the system being in the stable regime over 200 realizations in the random initial conditions of the mode amplitudes. The fixed parameters are the same as in Fig. 6.

where $a_{n, m}, b_{n, m}$, and $c_{n, m}$ are the modal amplitudes on the sites $\mathrm{A}, \mathrm{B}$, and $\mathrm{C}$ and in the $(n, m)$-th unit cell, respectively. $n$ and $m$ stand for the unit-cell index in the SSH lattice and quasi-1D lattice, respectively. $Z_{n, m}$ is the normalized excess carrier density in the active ring $\mathrm{A}, \tau_{p}$ and $\tau_{s}$ are the photon and spontaneous carrier lifetimes, respectively, $\alpha$ is the linewidth enhancement factor, $p_{A}$ is the normalized excess pump current in the ring $\mathrm{A}$, and $g_{B}$ and $g_{C}$ are the linear losses in the rings $\mathrm{B}$ and $\mathrm{C}$, respectively.

Here we show that a broad-area and phase-locked laser can be realized. The parameters are chosen similar to Refs. [18,31,32] and are typical for semiconductor lasers. The coupled-mode equations are integrated using random noise of field amplitudes between [0, 0.01] and equilibrium carrier density $Z_{n, m}=p_{A}$ as initial condition. The random noise as initial condition is chosen to trigger nonlinear behavior and see whether the mode is stable. Figure 6(a) shows that after a transient time, the system reaches a single laser mode regime. The laser mode is the topological zero-energy mode with $\operatorname{Im}(E)=0$. Figures 6(b) and 6(c) show that after a transient regime, only the zero-energy mode survives and reaches a steady state. The amplitudes of all the $A$ sites are equally distributed over the bulk and have a fixed $\pi$-phase difference. The laser system obtained is therefore broad area and phase locked.

The laser mode is topologically protected in addition to being broad area and phase locked. Figure 6(d) shows the spectrum of the system when a spatially varying and random asymmetric perturbation on the couplings, $\delta t_{1 \pm} \epsilon$ $\left[-0.12 t_{1}, 0.12 t_{1}\right]$, is added: $t_{1} e^{h} \rightarrow\left(t_{1}+\delta t_{1+}\right) e^{h}$ and $t_{1} e^{-h} \rightarrow$ $\left(t_{1}+\delta t_{1-}\right) e^{-h}$. This asymmetric perturbation accounts for perturbation in the coupling strength as well as for the imaginary gauge field. One can see that the zero-energy mode is still present. However, Figs. 6(e) and (f) show a slightly different behavior in the time series of the field amplitudes and the phase difference between the A rings. They do not

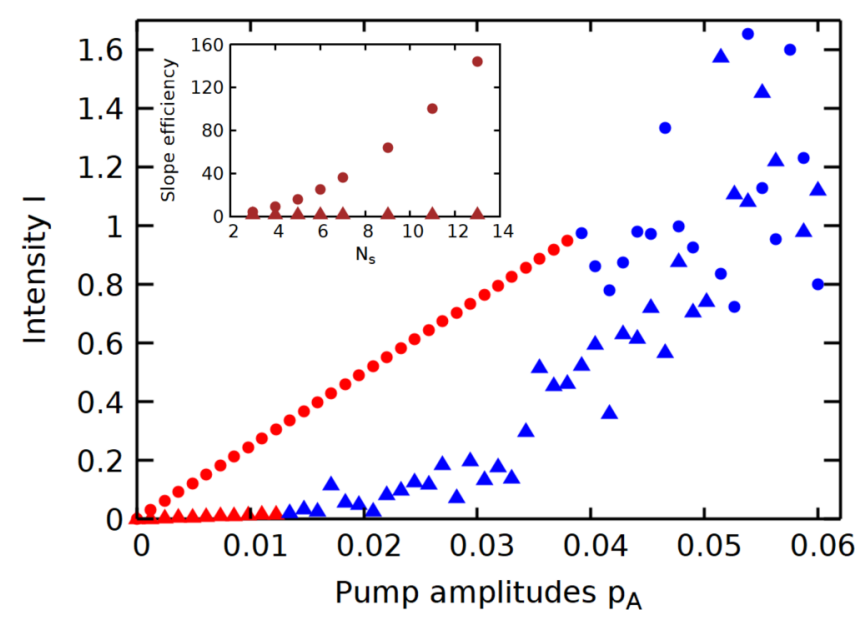

FIG. 8. Total intensity against pump amplitudes $p_{A}$ for the localized and delocalized topological mode with $N_{s}=9$. The triangles (circles) correspond to the topological edge (bulk) laser. The colors indicate if the system in the unstable (blue) or stable (red) regime. The inset shows the dependency of the slope efficiency with respect to $N_{s}$. The parameters are the same as in Fig. 6. 

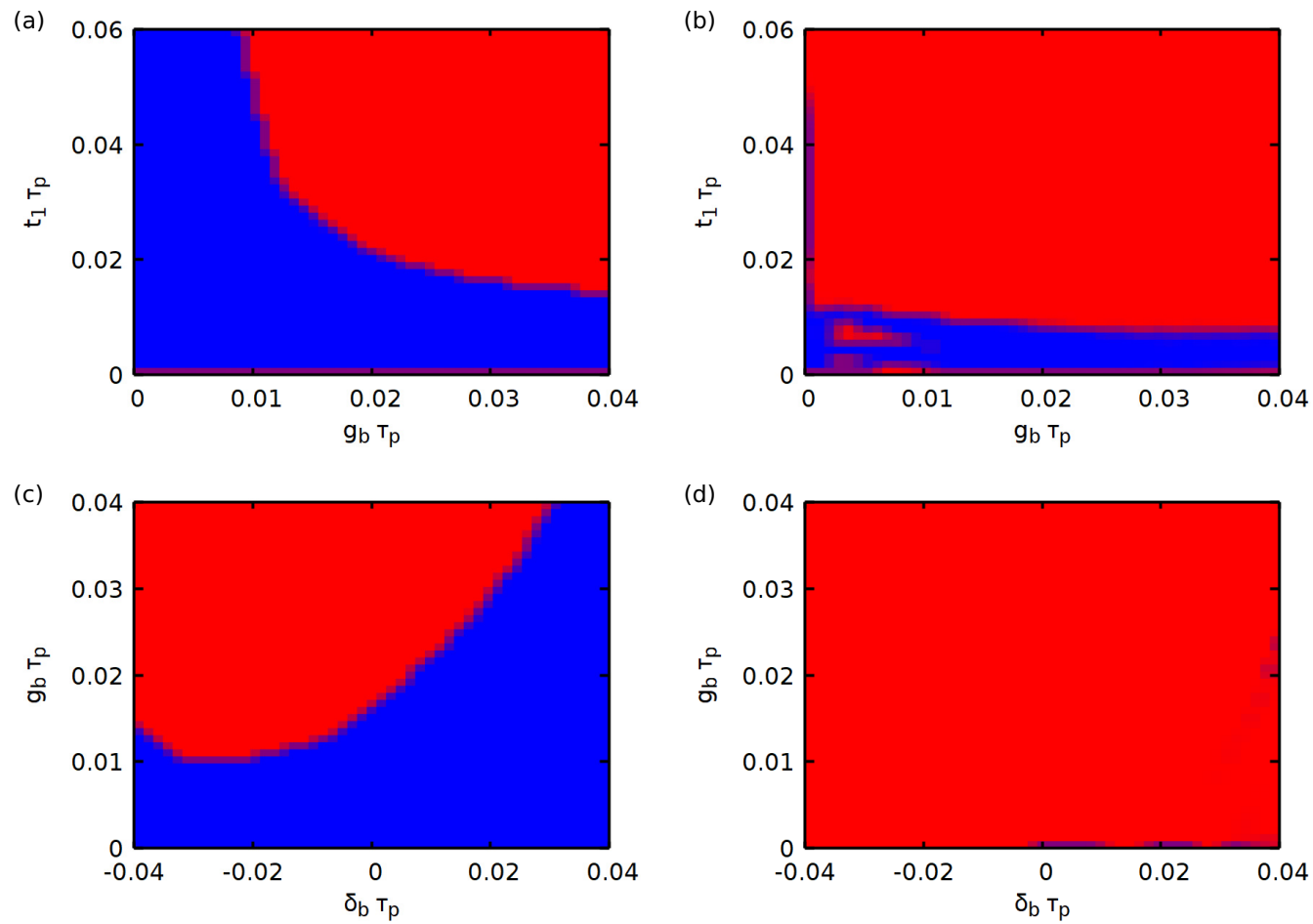

Probability of stable state

FIG. 9. Stability diagrams of the topological [(a), (c)] compact mode and [(b), (d)] extended mode. The color corresponds to the probability of the system being in the stable regime over 200 realizations in the random initial conditions of the mode amplitudes. The fixed parameters are the same as in Fig. 6 except here $p_{A}=0.01$.

reach the same values in amplitudes and phase differences. The amplitudes are not equally distributed but have slightly different offsets because of the different couplings between each sites: A single choice for the imaginary gauge field $h$ and $h^{\prime}$ cannot satisfy the $\left|r_{0, i}\right|=1$ conditions between each sites. Similarly, the phase differences are no longer equal to $\pi$ due to the nonlinear Henry factor $\alpha$. Nevertheless, here, the addition of perturbation in the coupling strengths does not give rise to unstable behavior in the amplitudes and phases of the topological extended mode.

The zero-energy mode shown in Fig. 6 does not suffer from nonlinear instabilities. Indeed, with the chosen parameters, the system reaches a stationary state which does not evolve into random oscillation in their amplitudes and phase differences. Even though the spatial stability of the topological mode may be guaranteed by its topological invariant, it is worth looking at its temporal stability in the parameter space to delimit the region where temporal instabilities arise due to the nonlinear terms. In the following, we will refer to the stable regime, the regime of single-mode lasing in the topological extended mode. Therefore, we say the system to be unstable (or stable) if oscillations are present (or not) in their amplitudes or phase differences.

Stable phase locking of the non-Hermitian gauge laser array is possible in a relatively large region of the parameter space. Figure 7 shows the stability diagram of the topological extended laser mode for different slices of the parameter space. The numerical results show the stability of the topological extended mode requires a minimum coupling strength [Figs. 7(a) and 7(b)]. One reason is that the $\mathcal{P} T$-symmetric phase of the SSH lattice is broken when the couplings are too small compared to the gain and loss [11]: The system is no longer in the single lasing mode regime. The second reason is that the nonreciprocal dissipative couplings need to be high enough in order to reach a (soft) synchronization [33]. On the other hand, instabilities arise when the detunings $\delta_{b}$ and $\delta_{c}$ are too high [Fig. 7(c)]. The major critical case is with positive detunings [Figs. 7(c) and 7(d)]. However, stability is retrieved if the dissipation is large enough to compensate the detunings in the rings $\mathrm{B}$ and $\mathrm{C}$.

Extended lasing modes present an important advantage in getting a better slope efficiency compared to the compact lasing mode. Figure 8 plots the total intensity, $I=\sum_{m, n}\left|\psi_{n, m}\right|^{2}$ against the pump amplitudes $p_{A}$ for the compact and extended lasing mode where the color plot corresponds to the system being in the stable or unstable regime. The numerical results show that the compact mode has lower slope efficiency compared to the extended mode. The remarkable difference is the scaling of the slope efficiency with the size of the system for the delocalized mode while it remains constant for the compact mode, as shown in the inset of Fig. 8. This is because of the extended nature of the mode whose contribution to the lasing intensity increases with the size of the system.

The imaginary gauge field helps to stabilize the system in the zero-energy lasing mode. The extended nature of the topological mode over the bulk allows the zero-energy mode to carry all the gain of the system while suppressing all the other bulk modes. The color plot in Fig. 8 indicates that the compact mode reaches an unstable regime for relatively low values of pump intensity whereas the extended mode is 


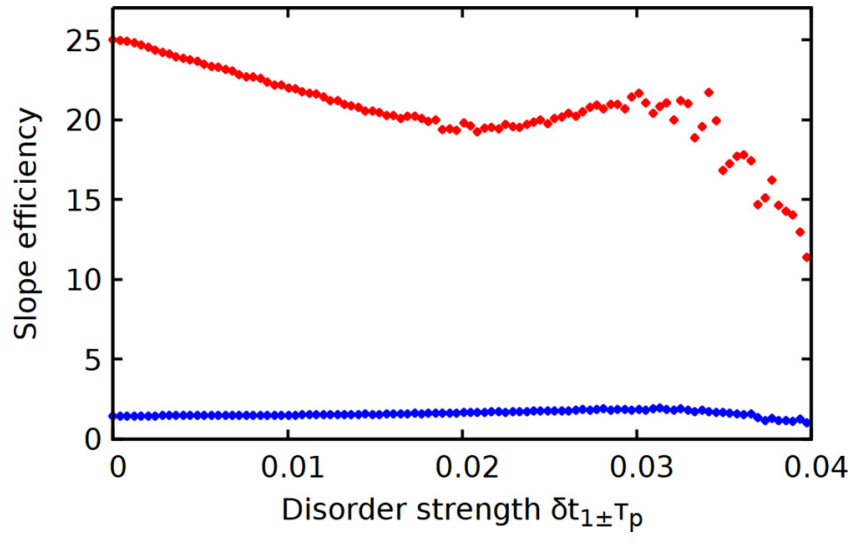

FIG. 10. Slope efficiency against disorder strength $\delta t_{1 \pm}$ (for $N_{s}=$ 9). The blue (red) dots correspond to the topological edge (extended) laser. For each disorder strength, the slope efficiency is average over 800 random realizations of the disorder. The parameters are the same as in Fig. 6.

unstable for higher pump intensities. The stability diagrams for the compact mode are shown in Figs. 9(a)-9(c). Compared to the extended mode in Figs. 9(b)-9(d), these diagrams demonstrate that extended modes have, indeed, larger regions of stability in the parameter space than compact modes.

Figure 10 shows the slope efficiency is robust against asymmetric disorder in the couplings, for both the compact and extended modes, due to the topological nature of the lasing mode. The compact mode has a relatively constant slope efficiency with increasing disorder strength. While the extended mode gives varying slope efficiency because of varying field distribution of the extended mode in the bulk as explained before, its slope efficiency remains higher compared to the compact mode. However, for high values of disorder strength in the couplings, the slope efficiency of the extended mode starts to decrease. This is explained because the delocalization of the topological mode, originating from nonreciprocal couplings, is highly perturbed by the asymmetric perturbation $\delta t_{1 \pm}$ in the couplings: The topological mode may not be completely delocalized anymore.

\section{CONCLUSION}

To summarize, we have shown a procedure to obtain topological modes extended over a $d$-dimensional bulk using an imaginary gauge field. In particular, we have demonstrated the existence of a topological extended mode in the kagome lattice in the rhombus geometry. This topological extended mode in the kagome lattice has been studied in the context of a lasing system where the laser rate equation is included to take into account nonlinear effects. By investigating its temporal stability, we proved that stable topological broad-area phase-locked laser operation is possible in a large region in the parameter space.

Furthermore, it has been shown that the topological extended mode presents clear advantages over the topological localized mode. The extended nature of the former topological mode over the bulk enhances its temporal stability and yields higher slope efficiency that scale with the size of the system.
In terms of footprint, a higher dimensional extended mode is also more advantageous compared to their lower dimensional one since at equivalent slope efficiency, for example, the system occupies a smaller region in real space when increasing the dimensionality of the system. This can lead to applications where transport of high energy density is possible. In addition, the lattice and material configurations are not limited to those studied in this paper. We believe that our work will provide a new route to achieving broad-area phase-locked high-power lasers.

\section{ACKNOWLEDGMENTS}

This research was undertaken using the supercomputing facilities at Cardiff University operated by Advanced Research Computing at Cardiff (ARCCA) on behalf of the Cardiff Supercomputing Facility and the HPC Wales and Supercomputing Wales (SCW) projects. We acknowledge the support of the SCW projects and Sêr Cymru II Rising Star Fellowship [80762-CU145 (East)], which are partly funded by the European Regional Development Fund (ERDF) via the Welsh Government.

\section{APPENDIX: EXTENDED TOPOLOGICAL MODE IN LIEB LATTICE AND 2D SSH LATTICE}

This Appendix provides additional examples about the delocalization of modes over a $d$-dimensional bulk: the Lieb lattice and the 2D SSH lattice.

\section{Lieb lattice}

This section focus on the anisotropic Lieb lattice [34], which is described by three sites by unit cell and intracell couplings $\left(t_{1}, t_{2}\right)$ and intercell couplings $\left(t_{3}, t_{4}\right)$ as shown in Fig. 11.

Specifically, we will delocalize the zero-energy corner mode present in the truncated Lieb lattice (see Fig. 12 for the finite size of the lattice). Figure 12(a) plots the normal-

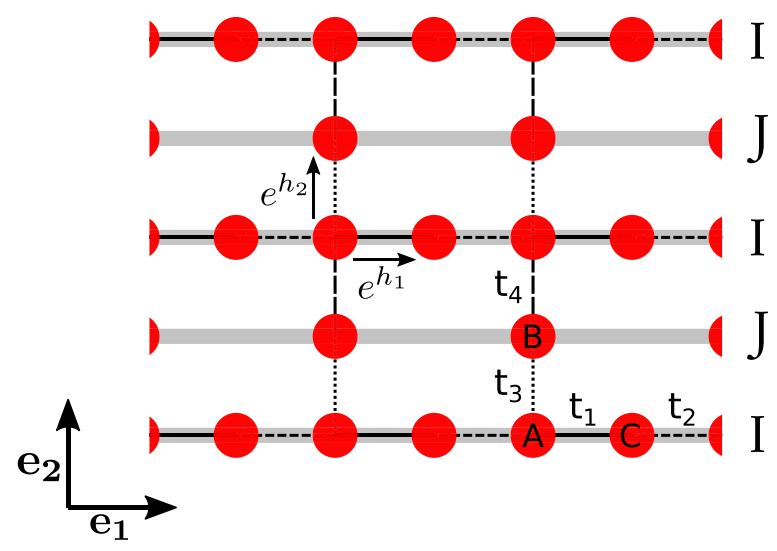

FIG. 11. Schematic of a non-Hermitian (anisotropic) Lieb lattice drawn in the quasi-1D lattice formalism. The solid and dotted lines represent the intracell couplings $t_{1}, t_{2}$, respectively. The short and long dashed lines denote the intercell couplings $t_{3}, t_{4}$, respectively. $e^{h_{1}}, e^{h_{2}}$ correspond to the imaginary gauge field. 
(a)

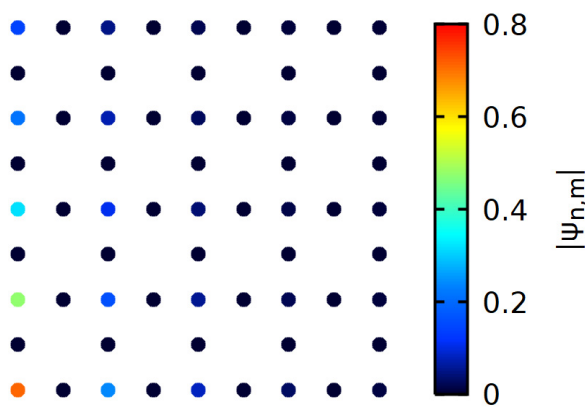

(b)

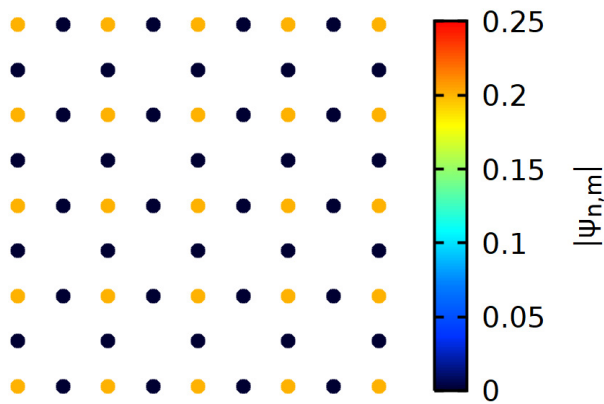

FIG. 12. The normalized field profile $\left|\psi_{n, m}\right|$ of the zero-energy mode of the truncated Lieb lattice is plotted in panel (a) for the Hermitian case and in panel (b) for the non-Hermitian case. Here, there are $N_{s}=9$ sites both in the $I$ lattice and the quasi-1D lattice. $t_{1}=0.02, t_{2}=0.06, t_{3}=0.03$, and $t_{4}=0.045$. The Hermitian setting corresponds to the case $h_{1}=h_{2}=0$, whereas the non-Hermitian setting is for $h_{1}=h_{0}, h_{2}=h_{0}^{\prime}$.

ized field distribution $\left|\psi_{n, m}\right|$ of the zero-energy corner mode obtained for $t_{1}<t_{2}$ and $t_{3}<t_{4}$.

Applying the recipe given in the main text to the (Hermitian) truncated Lieb lattice, we have $H_{I}=H_{\mathrm{SSH}}, H_{J}=$ $\operatorname{diag}\left(E_{B}, \ldots, E_{B}\right) . \tilde{H}_{I \leftarrow J}^{\dagger}=H_{I \leftarrow J}^{\dagger}=\left(t_{3}, \ldots, t_{3}\right)^{T}$ and $\tilde{H}_{J \rightarrow I}^{\dagger}=$ $H_{J \rightarrow I}^{\dagger}=\left(t_{4}, \ldots, t_{4}\right)^{T}$ are the $\left(N_{s} \times 1\right)$ matrices corresponding to the intra- and interstacked lattice couplings, respectively. $N_{s}$ is the number of sites on the $I$ lattices.

The zero-energy corner mode is analytically written as

$$
\psi_{I, m}^{(0)}=r_{0,2}^{m} \psi_{I, 0}^{(0)}
$$

with $\left[\psi_{I, 0}^{(0)}\right]_{n}=r_{0,1}^{n} a_{0,0}$ being the $n$th component of the zero-energy mode $\psi_{I, 0}^{(0)}$ of the SSH lattice, and where the interference conditions [Eq. (18) in the main text] give $r_{0,1}=-\frac{t_{1}}{t_{2}}$ and $r_{0,2}=-\frac{t_{3}}{t_{4}} \cdot a_{n, m}$ is the modal amplitude on the A site at the $n$th unit cell in the $m$ th lattice $I$.

We use an imaginary gauge field in order to delocalize the corner mode. Figure 11 sketches the gauge potential considered where $e^{h_{1}}$ and $e^{h_{2}}$ represent the phase factor in the couplings between the sites $\mathrm{A}$ and $\mathrm{C}$ and sites $\mathrm{A}$ and $\mathrm{B}$, respectively. The coupling matrices are then modified, and we have $\tilde{H}_{I \leftarrow J}^{\dagger}=\left(t_{3} e^{h_{2}}, \ldots, t_{3} e^{h_{2}}\right)$ and $\tilde{H}_{J \rightarrow I}^{\dagger}=$ $\left(t_{4} e^{-h_{2}}, \ldots, t_{4} e^{-h_{2}}\right)$. The interference conditions now yield

$$
r_{0,1}=-\frac{t_{1}}{t_{2}} e^{2 h_{1}}
$$

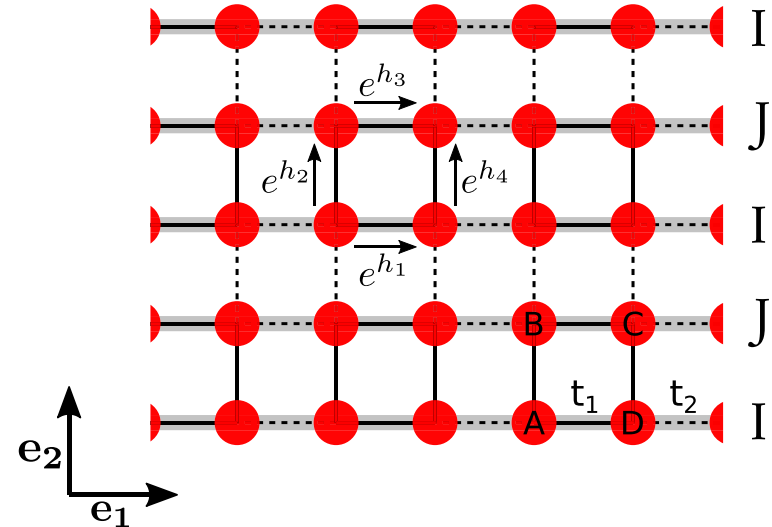

FIG. 13. Schematic of a non-Hermitian 2D SSH lattice drawn in the quasi-1D lattice formalism. The solid and dashed lines denote the intracell $\left(t_{1}\right)$ and intercell couplings $\left(t_{2}\right)$, respectively. $e^{h_{1}}, e^{h_{2}}, e^{h_{3}}$, and $e^{h_{4}}$ correspond to the imaginary gauge field.

and

$$
r_{0,2}=-\frac{t_{3}}{t_{4}} e^{2 h_{2}}
$$

Delocalization over the $\boldsymbol{e}_{\boldsymbol{i}}$ direction is achieved by requiring $\left|r_{0, i}\right|=1$, namely choosing

$$
h_{1}=h_{0}:=-\frac{1}{2} \ln \left(\frac{t_{1}}{t_{2}}\right)
$$

and

$$
h_{2}=h_{0}^{\prime}:=-\frac{1}{2} \ln \left(\frac{t_{3}}{t_{4}}\right) .
$$

Figure 12(b) show that the zero-energy mode is indeed extended over the whole bulk of the Lieb lattice.

\section{2D SSH lattice}

Here, we study the 2D SSH lattice [35], which is characterized by four sites by unit cell, and intracell $\left(t_{1}\right)$ and intercell $\left(t_{2}\right)$ couplings, as shown in Fig. 13. The method follows similarly to the two examples already provided previously.

We will delocalize the zero-energy mode present in the 2D SSH lattice [see Fig. 14(b) for the finite size of the lattice]. Figure 14(a) plots the normalized field distribution $\left|\psi_{n, m}\right|$ of the zero-energy corner mode which is exponentially localized on the bottom-left corner for $t_{1}<t_{2}$.

For the (Hermitian) truncated 2D SSH lattice, we have $H_{I}=H_{\mathrm{SSH}}, H_{J}=H_{\mathrm{SSH}}$. The intra- and interstacked lattice couplings are $\left(N_{s} \times 2\right)$ matrices given respectively by

$$
\tilde{H}_{I \leftarrow J}^{\dagger}=H_{I \leftarrow J}^{\dagger}=\left(\begin{array}{cc}
t_{1} & t_{1} \\
\vdots & \vdots \\
t_{1} & t_{1}
\end{array}\right)
$$

and

$$
\tilde{H}_{J \rightarrow I}^{\dagger}=H_{J \rightarrow I}^{\dagger}=\left(\begin{array}{cc}
t_{2} & t_{2} \\
\vdots & \vdots \\
t_{2} & t_{2}
\end{array}\right) .
$$

$N_{s}$ is the number of sites on the $I$ lattices. 
(a)

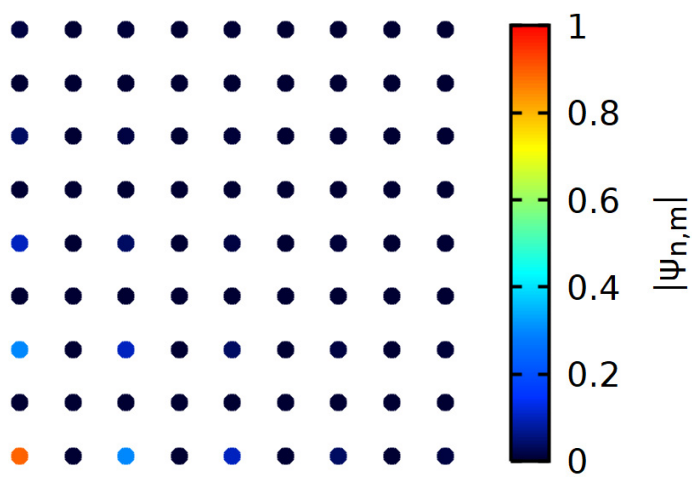

(b)

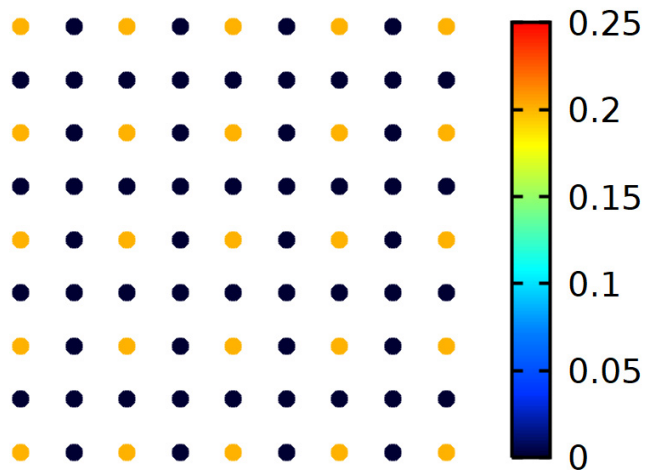

FIG. 14. The normalized field profile $\left|\psi_{n, m}\right|$ of the zero-energy mode of the truncated 2D SSH lattice is plotted in panel (a) for the Hermitian case and in panel (b) for the non-Hermitian case. Here, there are $N_{s}=9$ sites both in the $I$ lattice and the quasi-1D lattice. $t_{1}=0.02, t_{2}=0.06$. The Hermitian setting corresponds to the case $h_{1}=h_{2}=h_{3}=h_{4}=0$, whereas the non-Hermitian setting is for $h_{1}=h_{2}=h_{3}=h_{4}=h_{0}$.

The zero-energy corner can be written as

$$
\psi_{I, m}^{(0)}=r_{0,2}^{m} \psi_{I, 0}^{(0)}
$$

with $\left[\psi_{I, 0}^{(0)}\right]_{n}=r_{0,1}^{n} a_{0,0}$ being the $n$th component of the zeroenergy mode $\psi_{I, 0}^{(0)}$ of the SSH lattice where the interference conditions [Eq. (18) in the main text] give $r_{0,1}=-\frac{t_{1}}{t_{2}}$ and $r_{0,2}=-\frac{t_{1}}{t_{2}} \cdot a_{n, m}$ is the modal amplitude on the A site at the $n$th unit cell in the $m$ th lattice $I$.

We use an imaginary gauge field to change the localization property of the corner mode. Figure 13 sketches the gauge potential considered where $e^{h_{1}}, e^{h_{2}}, e^{h_{3}}$, and $e^{h_{4}}$ represent the phase factor in the couplings between the sites A and D, A and $\mathrm{B}, \mathrm{B}$ and $\mathrm{C}$, and $\mathrm{D}$ and $\mathrm{C}$, respectively. The values for $e^{h_{1}}, e^{h_{2}}, e^{h_{3}}$, and $e^{h_{4}}$ are not independent of each other. For the spectrum to remain unchanged, one need to satisfy

$$
h_{3}=h_{1}
$$

and

$$
h_{4}=h_{2} \text {. }
$$

With the imaginary gauge field, the coupling matrices are then modified. In particular, we have

$$
\tilde{H}_{I \leftarrow J}^{\dagger}=\left(\begin{array}{cc}
t_{1} e^{h_{2}} & t_{1} e^{h_{4}} \\
\vdots & \vdots \\
t_{1} e^{h_{2}} & t_{1} e^{h_{4}}
\end{array}\right)
$$

and

$$
\tilde{H}_{J \rightarrow I}^{\dagger}=\left(\begin{array}{cc}
t_{2} e^{-h_{2}} & t_{2} e^{-h_{4}} \\
\vdots & \vdots \\
t_{2} e^{-h_{2}} & t_{2} e^{-h_{4}}
\end{array}\right) .
$$

The interference conditions read

$$
r_{0,1}=-\frac{t_{1}}{t_{2}} e^{2 h_{1}}
$$

and

$$
r_{0,2}=-\frac{t_{1}}{t_{2}} e^{2 h_{2}}
$$

Delocalization over the $\boldsymbol{e}_{\boldsymbol{i}}$ direction is achieved by requiring $\left|r_{0, i}\right|=1$, namely choosing

$$
h_{1}=h_{2}=h_{0}:=-\frac{1}{2} \ln \left(\frac{t_{1}}{t_{2}}\right) .
$$

Figure 14(b) show that the zero-energy mode is indeed extended over the whole bulk of the 2D SSH lattice.
[1] T. Ozawa, H. M. Price, A. Amo, N. Goldman, M. Hafezi, L. Lu, M. C. Rechtsman, D. Schuster, J. Simon, O. Zilberberg, and I. Carusotto, Topological photonics, Rev. Mod. Phys. 91, 015006 (2019).

[2] A. B. Khanikaev, S. H. Mousavi, W.-K. Tse, M. Kargarian, A. H. MacDonald, and G. Shvets, Photonic topological insulators, Nat. Mater. 12, 233 (2013).

[3] S. Wong, M. Saba, O. Hess, and S. S. Oh, Gapless unidirectional photonic transport using all-dielectric kagome lattices, Phys. Rev. Research 2, 012011(R) (2020).

[4] M. C. Rechtsman, Y. Lumer, Y. Plotnik, A. Perez-Leija, A. Szameit, and M. Segev, Topological protection of photonic path entanglement, Optica 3, 925 (2016).

[5] S. Mittal, V. V. Orre, and M. Hafezi, Topologically robust transport of entangled photons in a 2D photonic system, Opt. Express 24, 15631 (2016).
[6] T. W. Hughes, M. Minkov, Y. Shi, and S. Fan, Training of photonic neural networks through in situ backpropagation and gradient measurement, Optica 5, 864 (2018).

[7] I. Liberal and N. Engheta, Near-zero refractive index photonics, Nat. Photon. 11, 149 (2017).

[8] X. Huang, Y. Lai, Z. H. Hang, H. Zheng, and C. T. Chan, Dirac cones induced by accidental degeneracy in photonic crystals and zero-refractive-index materials, Nat. Mater. 10, 582 (2011).

[9] M. Saba, J. M. Hamm, J. J. Baumberg, and O. Hess, Group Theoretical Route to Deterministic Weyl Points in Chiral Photonic Lattices, Phys. Rev. Lett. 119, 227401 (2017).

[10] G. Harari, M. A. Bandres, Y. Lumer, M. C. Rechtsman, Y. D. Chong, M. Khajavikhan, D. N. Christodoulides, and M. Segev, Topological insulator laser: Theory, Science 359, eaar4003 (2018). 
[11] M. Parto, S. Wittek, H. Hodaei, G. Harari, M. A. Bandres, J. Ren, M. C. Rechtsman, M. Segev, D. N. Christodoulides, and M. Khajavikhan, Edge-Mode Lasing in 1D Topological Active Arrays, Phys. Rev. Lett. 120, 113901 (2018).

[12] H. Schomerus, Topologically protected midgap states in complex photonic lattices, Opt. Lett. 38, 1912 (2013).

[13] H. Zhao, P. Miao, M. H. Teimourpour, S. Malzard, R. ElGanainy, H. Schomerus, and L. Feng, Topological hybrid silicon microlasers, Nat. Commun. 9, 981 (2018).

[14] K. Takata and M. Notomi, Photonic Topological Insulating Phase Induced Solely by Gain and Loss, Phys. Rev. Lett. 121, 213902 (2018).

[15] Y. Gong, S. Wong, A. J. Bennett, D. L. Huffaker, and S. S. Oh, Topological insulator laser using valley-Hall photonic crystals, ACS Photon. 7, 2089 (2020).

[16] B. Bahari, A. Ndao, F. Vallini, A. El Amili, Y. Fainman, and B. Kanté, Nonreciprocal lasing in topological cavities of arbitrary geometries, Science 358, 636 (2017).

[17] Z.-K. Shao, H.-Z. Chen, S. Wang, X.-R. Mao, Z.-Q. Yang, S.-L. Wang, X.-X. Wang, X. Hu, and R.-M. Ma, A high-performance topological bulk laser based on band-inversion-induced reflection, Nat. Nanotechnol. 15, 67 (2020).

[18] S. Longhi, Non-Hermitian gauged topological laser arrays, Ann. Phys. 530, 1800023 (2018).

[19] W. P. Su, J. R. Schrieffer, and A. J. Heeger, Solitons in Polyacetylene, Phys. Rev. Lett. 42, 1698 (1979).

[20] S. Longhi, Y. Kominis, and V. Kovanis, Presence of temporal dynamical instabilities in topological insulator lasers, EPL 122, 14004 (2018).

[21] S. Bittner, S. Guazzotti, Y. Zeng, X. Hu, H. Ylmaz, K. Kim, S. S. Oh, Q. J. Wang, O. Hess, and H. Cao, Suppressing spatiotemporal lasing instabilities with wave-chaotic microcavities, Science 361, 1225 (2018).

[22] N. Hatano and D. R. Nelson, Localization Transitions in Non-Hermitian Quantum Mechanics, Phys. Rev. Lett. 77, 570 (1996).

[23] F. K. Kunst, M. Trescher, and E. J. Bergholtz, Anatomy of topological surface states: Exact solutions from destructive interference on frustrated lattices, Phys. Rev. B 96, 085443 (2017).

[24] F. K. Kunst, G. van Miert, and E. J. Bergholtz, Boundaries of boundaries: A systematic approach to lattice models with solvable boundary states of arbitrary codimension, Phys. Rev. B 99, 085426 (2019).

[25] S. Mao, Y. Kuramoto, K.-I. Imura, and A. Yamakage, Analytic theory of edge modes in topological insulators, J. Phys. Soc. Jpn. 79, 124709 (2010).

[26] G. van Miert and C. Ortix, On the topological immunity of corner states in two-dimensional crystalline insulators, npj Quantum Mater. 5, 63 (2020).

[27] M. Ezawa, Higher-Order Topological Insulators and Semimetals on the Breathing Kagome and Pyrochlore Lattices, Phys. Rev. Lett. 120, 026801 (2018).

[28] A. El Hassan, F. K. Kunst, A. Moritz, G. Andler, E. J. Bergholtz, and $\mathrm{M}$. Bourennane, Corner states of light in photonic waveguides, Nat. Photon. 13, 697 (2019).

[29] L. Feng, Z. J. Wong, R.-M. Ma, Y. Wang, and X. Zhang, Singlemode laser by parity-time symmetry breaking, Science 346, 972 (2014).

[30] M. A. Bandres, S. Wittek, G. Harari, M. Parto, J. Ren, M. Segev, D. N. Christodoulides, and M. Khajavikhan, Topological insulator laser: Experiments, Science 359, eaar4005 (2018).

[31] H. G. Winful and L. Rahman, Synchronized Chaos and Spatiotemporal Chaos in Arrays of Coupled Lasers, Phys. Rev. Lett. 65, 1575 (1990).

[32] R.-d. Li and T. Erneux, Preferential instability in arrays of coupled lasers, Phys. Rev. A 46, 4252 (1992).

[33] J. Ding, I. Belykh, A. Marandi, and M.-A. Miri, Dispersive Versus Dissipative Coupling for Frequency Synchronization in Lasers, Phys. Rev. Appl. 12, 054039 (2019).

[34] D. Guzmán-Silva, C. Mejía-Cortés, M. A. Bandres, M. C. Rechtsman, S. Weimann, S. Nolte, M. Segev, A. Szameit, and R. A. Vicencio, Experimental observation of bulk and edge transport in photonic Lieb lattices, New J. Phys. 16, 063061 (2014).

[35] F. Liu and K. Wakabayashi, Novel Topological Phase with a Zero Berry Curvature, Phys. Rev. Lett. 118, 076803 (2017). 